\title{
COMMUTING VECTORFIELDS ON OPEN MANIFOLDS ${ }^{1}$
}

\author{
BY J. WOLFGANG SMITH
}

Communicated by Norman Steenrod, March 24, 1969

Let $M$ be an open orientable differentiable $n$-manifold. More precisely, we will take $M$ and vectorfields over $M$ to be of class $C^{\infty}$. A nonzero vectorfield $X$ on $M$ will be called nonrecurrent if the 1-dimensional foliation associated with $X$ is regular (see [4, Chapter I]) and admits no compact leaves. The notation $H^{p}(M ; Z)=Q$ shall mean that the $p$-dimensional singular integral cohomology of $M$ is trivial or admits no torsion of order 2, depending on whether $p$ is even or odd, respectively.

THEOREM 1. Let $X$ be a nonrecurrent vectorfield on $M$ and let $A \subset M$ be relatively compact. When $H^{n-1}(M ; Z)=Q$ there exists a vectorfield $Y$ on $A$ such that $X, Y$ are linearly independent and commute.

TheOREM 2 . When $H^{n-1}(M ; Z)=Q$ every relatively compact subset of $M$ submerges in the plane.

For $n>4$ Theorem 2 is implied by a result of I. M. James and E. Thomas (quoted as Theorem 8.6 in [5]). Moreover, we note that the cohomological triviality condition is crucial to both Theorems 1 and 2. A very simple example shows this in the case of Theorem 1: Let $M$ be Euclidean 3-space with a point 0 removed and let $X=\partial / \partial r$, where $r$ denotes distance to 0 . Let $S$ denote the unit sphere centered at 0 and let $\pi: M \rightarrow S$ denote radial projection. There exist relatively compact subsets $A \subset M$ such that $\pi(A)=S$. A vectorfield $Y$ on $A$ which commutes with $X$ induces then a vectorfield $\bar{Y}$ on $S$ such that $\bar{Y}$ pulls back to $Y$ under $d \pi$. Moreover, if $(X, Y)$ are linearly independent, $\bar{Y}$ must be nonzero, showing that the conclusion of Theorem 1 does not hold in this case. It is also possible to display examples of open orientable $C^{\infty}$-manifolds $M$ with relatively compact $A \subset M$ which do not submerge in the plane. We may take $M$ to be the punctured real projective space of dimension 5 , for instance. It is known [5, p. 201] that this space does not submerge in the plane. But obviously $M$ admits relatively compact subsets $A$ which are in fact diffeomorphic to $M$.

In this note we shall derive Theorems 1 and 2 from results established in [6]. First a few definitions: If $F$ is a regular orientable $p$ -

\footnotetext{
1 Research supported in part by National Science Foundation Grant GP-6648.
} 
dimensional foliation on $M$, we let $M / F$ denote the quotient space obtained by identifying points of $M$ belonging to the same leaf of $F$. Regularity of $F$ implies that $M / F$ can be regarded as a differentiable manifold. It will be orientable, but in general non-Hausdorff. Let $\chi_{F}$ denote its Euler class (the algebraic sign of $\chi_{F}$ being determined by a choice of orientation). The foliation $F$ is said to extend on $A$ ( $A$ being a subset of $M$ ) provided there exists an orientable $(p+1)$ dimensional foliation $\hat{F}$ on $A$ with $F \subset \hat{F}$.

Theorem A. An orientable regular foliation $F$ on $M$ with $\chi_{F}=0$ extends on relatively compact subsets of $M$.

When $M / F$ is Hausdorff, this conclusion follows easily by classical obstruction theory. In the general case it does not. An essential ingredient in the proof is a triangulation theorem contributed by J. R. Munkres, which appears to be also of independent interest (see [6, Appendix]). The next step is

LEMMA B. Let $F$ be a regular 1-dimensional foliation on $M$ without compact leaves. The natural projection $\pi: M \rightarrow M / F$ induces then an isomorphism between the respective singular homology groups.

We refer to [6] for proofs of Theorem A and Lemma B. Combining these results and noting that $\chi_{F}$ has order 2 when $n$ is even (see $[3, \mathrm{p}$. 41]) we obtain what will be needed:

EXTENSION THEOREM. Let $F$ be a regular orientable 1-dimensional foliation on $M$ without compact leaves. If $H^{n-1}(M ; Z)=Q$, then $F$ extends on relatively compact subsets of $M$.

We proceed to establish Theorem 1 . Let $X$ be a nonrecurrent vectorfield on $M$ and $A \subset M$ an open relatively compact subset. Let $B$ denote a compact neighborhood of $A$ and let $F$ denote the foliation associated with $X$. If $H^{n-1}(M ; Z)=Q$, then by the Extension Theorem there exists an orientable 2-dimensional foliation $\hat{F}$ on $B$ with $F \subset \hat{F}$. Using a Riemannian metric on $M$ one obtains a differentiable field $Z$ of unit vectors on $B$ such that $Z$ is orthogonal to $X$ and $(X, Z)$ span $\hat{F}$. But this implies that $[X, Z]=a X+b Z$ where $a, b$ are differentiable functions on $B$. Let $Y=\alpha X+\beta Z$, where $\alpha, \beta$ are smooth functions on $B$. An easy calculation shows that the condition $[X, Y]$ $=0$ is equivalent to the differential equations

$$
\begin{aligned}
& \langle X, d \beta\rangle+\beta b=0 \\
& \langle X, d \alpha\rangle+\beta a=0
\end{aligned}
$$


where $\langle$,$\rangle denotes the inner product. It remains to be shown that$ this system admits a solution on $A$ with $\beta>0$. But this can be accomplished by the classical theory of characteristics for first order equations (see for instance [1, Chapter 2]). Equations (1) and (2) both have the integral curves of $X$ as their characteristics. The problem is thus reduced to integrating the ordinary differential equations

$$
\begin{aligned}
& d \beta / d s+\beta b=0 \\
& d \alpha / d s+\beta a=0
\end{aligned}
$$

along the integral curves of $X$, where $s$ denotes a parameter. To construct a solution, we cover $A$ by a finite sequence of "tubes" $T_{1}, \cdots, T_{r}$; each $T_{i}$ being determined by a local cross-section $S_{i}$ to the characteristics. More precisely, $S_{i}$ is taken to be a closed disc of dimension $n-1$ smoothly imbedded in $B$ which meets each integral curve of $X$ in at most one point (which is possible by nonrecurrence of $X$ ). The tube $T_{i}$ is then taken to be the set of all points $x \in B$ which can be connected to $S_{i}$ by an integral curve of $X$ lying entirely in $B$. We note that each $T_{i}$ is a closed subset of $B$. One can prescribe $\beta, \alpha$ on $S_{1}$, and this determines $\beta, \alpha$ on $T_{1}$ by integrating equations (1*) and $\left(2^{*}\right)$, respectively. We note also that $\beta>0$ on $S_{1}$ implies $\beta>0$ on $T_{1}$. Let us assume that $\beta, \alpha$ have been determined on $T_{1}, \cdots, T_{j}$ for some $j<r$ so as to be consistent on the intersections and such that $\beta>0$. The values of $\beta, \alpha$ are then prescribed on $S_{j+1} \cap T_{1} \cap \cdots \cap T_{j}$, which constitutes a closed subset of $S_{j+1}$. The given functions can be smoothly extended to $S_{j+1}$, preserving $\beta>0$. Integrating Equations $\left(1^{*}\right)$ and $\left(2^{*}\right)$ over $T_{j+1}$ with the given initial values gives an extension of $\beta, \alpha$ to $T_{j+1}$. The construction is thus completed in $r$ steps.

To prove Theorem 2 one first observes that since $M$ is open it submerges in the real line (by Theorem 4.7 in M. Hirsch [2]). Given a Riemannian metric, such a submersion determines a nonrecurrent vectorfield $X$ on $M$ (i.e. the gradient of the submersion). If

$$
H^{n-1}(M ; Z)=Q
$$

and $A$ is an open relatively compact subset of $M$, then by Theorem 1 (or equally well, by the Extension Theorem) there exists a vectorfield $Y$ on $A$ such that $X, Y$ are linearly independent. By Theorem B in Phillips [5] this implies that $A$ submerges in the plane.

\section{REFERENCES}

1. R. Courant and D. Hilbert, Methods of mathematical physics. Vol. II, Interscience, New York, 1962. 
2. M. Hirsch, On embedding differentiable manifolds in Euclidean space, Ann. of Math. (2) 73 (1961), 566-571.

3. J. W. Milnor, Lectures on characteristic classes, Mimeographed notes, Princeton University, Princeton, N.J., 1957.

4. R. S. Palais, $A$ global formulation of the Lie theory of transformation groups, Mem. Amer. Math. Soc. No. 22, 1957.

5. A. Phillips, Submersions of open manifolds, Topology 6 (1966), 171-206.

6. J. W. Smith, Extending regular foliations, Ann. Inst. Fourier (Grenoble) (to appear).

Oregon State University, Corvallis, Oregon 97331 